\title{
Usando a metacognição para analisar um caso de erro diagnóstico em simulação de alta fidelidade
}

\author{
Using metacognition to analyze a misdiagnosis case in high-fidelity simulation
}

\author{
Mauricio Abreu Pinto Peixoto' \\ Marcos Antônio Gomes Brandão' \\ Gerson Alves Pereira Junior ${ }^{2}$ \\ Juliana Faria Campos ${ }^{1}$ \\ Jaqueline da Silva Soares Souto ${ }^{1}$ \\ geac.ufrj@gmail.com \\ marcosbrandao@ufrj.br \\ gersonapj@gmail.com \\ jujufariacampos@yahoo.com.br \\ jaquelinesouto91@gmail.com
}

\section{RESUMO}

Introdução: As equipes médicas atuam constantemente diante de pacientes em estado crítico e ambientes complexos. Nesses ambientes, entende-se que processos cognitivos, metacognitivos e afetivos coexistem, de modo a propiciar ou impedir um desempenho adequado ${ }^{1,2}$. Nesta pesquisa, analisa-se um caso de erro diagnóstico sob a perspectiva metacognitiva.

Objetivos: Este estudo teve como objetivos descrever os processos de pensamento que levaram ao erro e investigar possíveis contribuições dos processos metacognitivos para o ensino médico.

Métodos: Fez-se uma entrevista em grupo ${ }^{3}$ com a equipe vencedora de uma olimpíada de simulação de atendimento a pacientes críticos realizada em um congresso nacional de educação médica. Adotou-se a análise de conteúdo ${ }^{4}$, codificada por Atlas-ti@, segundo Efklides ${ }^{5}$, seguida da extração das categorias empíricas no editor de mapas mentais SimpleMind@. O estudo foi registrado com CAAE n० 96007018.5.0000.5286 e aprovado (Parecer n².938.945) pelo Comitê de Ética em Pesquisa do Instituto de Estudos e Saúde Coletiva da Universidade Federal do Rio de Janeiro (UFRJ).

Resultados: A equipe, antes da olimpíada, previu cenários possíveis. Durante a competição, o cenário simulado apresentado era semelhante a um dos esperados. Observou-se então que a equipe, sem se dar conta, enviesou todo o seu raciocínio visando confirmar o diagnóstico previsto a priori. São descritos os vários mecanismos metacognitivos envolvidos nesse processo. A equipe possuía conhecimento suficiente para estabelecer o diagnóstico correto, mas não o fez por distorção dos processos de pensamento. Esse caso ilustra o fato de que, para praticar medicina, conhecimento não é suficiente; aprender a pensar também é necessário. Ademais, estabelece-se uma proposta de quadro teórico, em que a simulação se apresenta como metodologia problematizadora, fornecendo um contexto no qual a metacognição e o Arco de Maguerez ${ }^{6}$ integramse harmonicamente com a Teoria da Aprendizagem Significativa de Ausubel ${ }^{7,8}$ para o desenvolvimento da competência profissional ${ }^{6}$.

Conclusão: A metacognição permite elucidar eventos como os aqui descritos, sugerindo também que seu ensino e sua prática poderiam contribuir para a redução do erro médico.

Palavras-chave: Metacognição; Aprendizagem; Estudantes; Treinamento por Simulação; Erros de Diagnóstico; Tomada de Decisão Clínica.

\begin{abstract}
Introduction: Medical teams constantly work with patients in critical conditions and complex environments. Within these environments, it is understood that cognitive, metacognitive and affective processes coexist, promoting or preventing an adequate performance ${ }^{1,2}$. In this study, a case of medical misdiagnosis is analyzed from a metacognitive perspective.

Objectives: 1-Describe the thinking processes that led to the misdiagnosis, 2-Investigate possible contributions of metacognitive processes to medical education.

Methods: Group interview ${ }^{3}$ with the winning team of a simulation contest for attending critical patients held at a national medical education congress. Content analysis ${ }^{4}$, coded by Atlas-ti ${ }^{\mathrm{TM}}$, according to Efklides ${ }^{5}$, followed by the extraction of empirical categories in the SimpleMind ${ }^{\mathrm{TM}}$ Mind Map Editor. The study was registered (CAAE 96007018.5.0000.5286) and approved (Opinion No. 2,938,945) by the Research Ethics Committee of Institute of Collective Health Studies at the Federal University of Rio de Janeiro.

Results: Before the contest, the team predicted possible scenarios. During the contest, the simulated scenario presented to the participants was similar to one of the previously predicted scenarios. It was then observed that the team unconsciously biased all their reasoning aiming to confirm the previously predicted diagnosis. Different metacognitive mechanisms involved in this process are described. The team had sufficient knowledge to establish the correct diagnosis but did not do it due to the distortion of their thinking processes. This case illustrates the fact that, to practice medicine, knowledge is not enough; learning to think is also necessary. In addition, a proposal for a theoretical framework is established, where the simulation presents itself as a problematizing methodology, providing a context where metacognition and the Maguerez Arch ${ }^{6}$ are harmoniously integrated with Ausubel's Meaningful Learning Theory ${ }^{7,8}$ for professional competence ${ }^{6}$ development.
\end{abstract}

Conclusions: It is concluded that metacognition can elucidate events such as those described here, also suggesting that its teaching and practice could contribute to the reduction in medical misdiagnosis.

Keywords: Metacognition; Learning; Students; Simulation Training; Diagnostic Errors; Clinical Decision-Making.

${ }^{1}$ Universidade Federal do Rio de Janeiro, Rio de Janeiro, Rio de Janeiro, Brasil.

2 Universidade de São Paulo, São Paulo, São Paulo, Brasil.

Editora-chefe: Daniela Chiesa

| Editora associada: Daniela Chiesa

Recebido em 13/07/20; Aceito em 11/03/21. | Avaliado pelo processo de double blind review. 


\section{INTRODUÇÃO}

As equipes médicas atuam constantemente diante de pacientes em estado crítico e ambientes complexos. Nesses ambientes, entende-se que processos cognitivos, metacognitivos e afetivos coexistem, de modo a propiciar ou impedir um desempenho adequado ${ }^{1,2}$. Durante as emergências médicas, eventos adversos e erros potencialmente fatais comumente ocorrem. Pesquisas revelaram que há vários erros $\operatorname{cognitivos}^{9}$ na tomada de decisões clínicas que podem ocorrer devido à incerteza diagnóstica, à novidade do caso e à frequência de distrações específicas de emergências médicas, como também podem ocorrer como resultado de dificuldades de comunicação ${ }^{10,11}$.

A simulação realística é uma metodologia ativa de ensino-aprendizagem que facilita a capacitação, aproximandose do cenário real ${ }^{12}$. Apesar dos potenciais benefícios dos treinamentos utilizando simulação, há necessidade de mais pesquisas para examinar a natureza da aprendizagem e do desempenho em ambientes simulados ${ }^{13-15}$.

Diante desse contexto, acredita-se que a simulação realística, por meio de cenários simulados, seja capaz de explorar as habilidades e experiências metacognitivas de médicos, proporcionando ganhos em lidar com a complexidade da tarefa diagnóstica, diante das incertezas dos casos, hipóteses e alternativas de decisão.

Assim, procuramos abordar as seguintes questões de pesquisa:

- Qual é a natureza dos processos metacognitivos de uma equipe de estudantes de Medicina que enfrenta dificuldades com uma simulação de emergência médica?

- Que contribuições os processos metacognitivos podem trazer para o ensino de medicina?

A metacognição pode contribuir para esse processo, já que é entendida como um conhecimento sobre os próprios processos e produtos cognitivos. Devido à escassez de pesquisas nessa área, o presente estudo buscou atingir dois objetivos. Em primeiro lugar, visou explicar o insucesso de uma equipe de estudantes de Medicina no atendimento de uma de simulação de emergência médica descrevendo e interpretando a natureza dos seus processos metacognitivos. Em segundo lugar, feito isso, procurou estabelecer eventuais contribuições desses processos para o ensino de medicina.

\section{MÉTODOS}

\section{Cenário}

A simulação ocorreu em uma "olimpíada de simulação de atendimento a pacientes críticos", em um congresso nacional de educação médica. Essa olimpíada tem sido um evento précongresso regular da Associação Brasileira de Educação Médica (Abem). Nele são utilizados cenários de simulação clínica, tendo como base a construção das estações do Exame Nacional de Revalidação de Diplomas Médicos Expedidos por Instituição de Educação Superior Estrangeira (Revalida). Esses cenários são elaborados por docentes das escolas médicas das cidades do congresso, orientados por outro docente do programa de simulação da associação nacional. Foram disponibilizadas inscrições para que estudantes de Medicina, de forma voluntária, se organizassem em equipes de atendimento para a participação na olimpíada.

Para a simulação, utilizaram-se um manequim de alta fidelidade e o ambiente físico. Os equipamentos foram devidamente estruturados e disponibilizados para atender a uma situação de emergência, apresentando a complexidade e fidelidade para tal. O manequim é disponibilizado por empresa que participa do congresso em parceira com a Abem.

Para a avaliação do desempenho das equipes de estudantes inscritos, havia uma comissão julgadora, composta por três docentes, que assistia ao atendimento simulado realizado. Dessa forma, foi possível observar os eventos e controlar a duração do cenário (igual para todos os cenários e equipes), assim como, eventualmente, determinar o seu encerramento precoce. Havia também um facilitador, que permanecia dentro do ambiente simulado, sendo responsável por atender às demandas da equipe, como solicitações de exames complementares, informações não apresentadas pelo simulador, entre outras.

Duas equipes diferentes de estudantes competiam na mesma estação simulada, e a primeira das equipes ficava confinada (sem comunicação), não tendo assistido ao desempenho da outra equipe. Solicitou-se às equipes participantes que fizessem todas as comunicações em voz alta e enunciassem as hipóteses diagnósticas, os raciocínios e as razões que justificavam as diferentes condutas e procedimentos, assim como quaisquer solicitações feitas. O principal critério de julgamento foi o acerto na hipótese diagnóstica e sua correspondente terapêutica. Terminado o cenário, a equipe, conforme o seu desempenho, poderia ser eliminada da competição ou prosseguir para os cenários seguintes, elaborados com grau crescente de complexidade a cada fase.

As estações simuladas foram públicas e ocorreram em duas salas, onde, em adição aos componentes já citados, havia lugares para plateias compostas pelas demais equipes participantes e por outros espectadores, geralmente congressistas de alguma forma ligados ao tema ou aos membros das equipes. 


\section{Descrição do caso em estudo}

Para este estudo, selecionou-se intencionalmente a equipe vencedora da olimpíada. Por falhar na realização do diagnóstico médico e, consequentemente, no seu tratamento, ela foi eliminada ao participar do primeiro cenário simulado. No entanto, a equipe conseguiu voltar à competição pela oportunidade que teve na repescagem e, ao final, consagrou-se a vencedora da olimpíada. Então, é interessante explicar como uma equipe capaz o suficiente para vencer falha logo no início.

Neste estudo, somente será analisado o desempenho da equipe no atendimento do primeiro caso, em que ocorre a falha no diagnóstico. $\mathrm{O}$ caso clínico apresentado à equipe consistia em uma mulher com quadro de tromboembolismo pulmonar (TEP). No entanto, a equipe realizou o diagnóstico de síndrome coronariana aguda e fez todas as intervenções pertinentes para o seu tratamento.

Os participantes do estudo foram os componentes da equipe vencedora da olimpíada, composta por quatro estudantes de Medicina de uma faculdade privada, cursando o oitavo período, com faixa etária entre 23 e 26 anos. Para fins deste estudo, a capacidade da equipe foi considerada adequada por conta do seu nível de graduação e pelo fato de, em iguais condições, ter superado todas as outras concorrentes.

\section{Tipo de estudo}

Trata-se de um estudo de caso, com desenho qualitativo, orientado para a descrição de eventos que levaram à falha no diagnóstico de estudantes de Medicina durante participação em cenários simulados de cuidados críticos. $O$ estudo foi cadastrado no Comitê de Ética em Pesquisa do Instituto de Estudos de Saúde Coletiva da Universidade Federal do Rio de Janeiro (UFRJ) sob Certificado de Apresentação para Apreciação Ética (CAAE) no 96007018.5.0000.5286 e aprovado pelo Parecer $\mathrm{n}^{\circ} 2.938 .945$, datado de 4 de outubro de 2018.

No projeto aprovado, entre outras, foi explicitada a intenção de utilizar o congresso como ambiente do estudo, assim como a descrição pormenorizada dos aspectos éticos e metodológicos. A Abem e as comissões do congresso nacional de educação médica tiveram ciência prévia e aprovaram a realização do estudo. De modo a preservar o anonimato dos sujeitos, os nomes originais foram substituídos por pseudônimos.

\section{Coleta dos dados}

A olimpíada de simulação foi realizada como um evento pré-congresso, e fez-se a entrevista, com os resultados já públicos, no último dia. Como técnica de coleta, adotou-se a entrevista em grupo ${ }^{3}$, que foi gravada e transcrita. Realizouse a entrevista em ambiente confortável e privativo, e as perguntas foram dirigidas, na maior parte das vezes, para todos indistintamente. Em alguns casos, visando a esclarecimentos específicos, era dirigida uma questão a um sujeito em particular.

O entrevistador não fez uso de um rol de perguntas previamente preparadas. Preferiu orientar-se por um roteiro intencionalmente vago (Quadro 1). Em seguida, ao sabor do rumo indicado pelos participantes, o entrevistador, dirigido pelo foco do estudo, foi progressivamente fazendo outras questões que procuravam, às vezes, detalhar eventos e, às vezes, explicitar pensamentos. Dessa forma, dirigiu seus questionamentos buscando desvelar, do ponto de vista metacognitivo, os processos de pensamento que modularam o desempenho da equipe, levando-a à falha no diagnóstico e à sua consequente eliminação precoce. Mais especificamente, procurou entender por que ela foi eliminada logo no primeiro cenário, dado que, por conta da repescagem, pôde continuar na competição, mostrando-se suficientemente capaz para vencê-la.

\section{Análise dos dados}

Para a análise de conteúdo ${ }^{4}$, utilizou-se a teoria metacognitivista ${ }^{5,16}$. Metacognição é:

[...] um termo amplo, usado para descrever diferentes aspectos do conhecimento que construímos sobre como nós percebemos, recordamos, pensamos e agimos. Uma capacidade de saber sobre o que sabemos. [...] Sendo, portanto, um discurso de segundo nível sobre o conhecimento, caracteriza-se como um sistema de pensamento focado sobre a atividade cognitiva humana (p. 69-70) ${ }^{16}$

A entrevista foi submetida à técnica de análise de conteúdo ${ }^{4}$ em quatro etapas sequenciais. Na primeira etapa, a entrevista foi gravada e transcrita para arquivo-texto. $\mathrm{Na}$

Quadro 1. Roteiro da entrevista

\section{Etapas a serem detalhadas durante a entrevista}

- Apresentação pessoal e da equipe de pesquisa.

- Apresentação do estudo e formalização dos aspectos éticos.

- Solicitação de anuência ao TCLE.

- Apresentação e identificação dos sujeitos e sua anuência ao TCLE.

- Expectativas relativas à olimpíada.

- Características e formação da equipe.

- Comportamento geral da equipe durante a olimpíada.

- Detalhamento das circunstâncias e dos eventos associados ao erro diagnóstico.

- Avaliação dos participantes relativa à participação deles na olimpíada.

Fonte: Elaborado pelos autores 
segunda, de leitura flutuante, o texto foi lido repetidamente pelo entrevistador para entender o sentido global dos eventos, assim como os papéis particulares dos sujeitos e suas relações no grupo.

$\mathrm{Na}$ terceira etapa, o texto foi codificado com o software de análise qualitativa Atlas-ti@, segundo as variáveis metacognitivas de Efklides $^{5}$, entendidas neste estudo como categorias teóricas. Nessa etapa, os sentidos globais percebidos antes foram sendo traduzidos em termos de variáveis metacognitivas. Assim, ao final dessa etapa, havia uma listagem de todos os trechos que evidenciavam atividade metacognitiva, codificados pela variável metacognitiva correspondente.

Na quarta etapa, buscou-se, então, responder à questão norteadora da pesquisa. Usando-se o editor de mapas mentais SimpleMind $\odot$, o material produzido foi ordenado em dois passos.

No primeiro, as partes dos discursos expressando atividade metacognitiva foram ordenadas cronologicamente. Dessa forma, foi possível mostrar um processo que, partindo de um diagnóstico inicial, prosseguiu com evolução desfavorável do caso até o incidente crítico que, por fim, sinalizou a falha para a equipe.

O segundo relacionou cada momento dessa cronologia à variável metacognitiva correspondente, que fora identificada na terceira etapa. Ao fazê-lo, pudemos descrever, na perspectiva metacognitiva, o processo de pensamento que explicou por que uma equipe capaz falhou onde não era esperado.

O desfecho primário analisado foi a presença de eventos metacognitivos no discurso dos sujeitos da pesquisa. Já o desfecho secundário foi a demonstração/descrição de um papel significativo dos processos metacognitivos no desempenho das equipes participantes da olimpíada.

\section{RESULTADOS E DISCUSSÃO}

Os resultados a seguir serão descritos em texto. Como já mencionado, no primeiro cenário, a equipe, ante um quadro de TEP, estabeleceu um diagnóstico equivocado de infarto agudo do miocárdio (IAM). Para entender o processo que levou ao erro, é necessário olhar para um tempo anterior à olimpíada.

Começa quando o grupo se organiza para dela participar, e o faz de forma séria e capaz. O processo de escolha dos participantes e composição da equipe é motivo de outro estudo futuro.

O que aqui importa é que, tendo se constituído como equipe, o grupo avaliou as condições e regras da olimpíada e, preliminarmente, previu um conjunto de cenários possíveis. Entre eles, havia o IAM, o que faz sentido, dado que competição seria em atendimento a pacientes críticos.

É o momento em que podemos identificar a utilização da Habilidade Metacognitiva ${ }^{(a)}(\mathrm{HM})$ de previsão ${ }^{(b)}$. Assim:
[Mariana] Desde o começo a gente estava pensando assim...

[Francisco] O que poderia chegar para gente...

[Mariana] Agente já estava com síndrome coronariana na cabeça.

Feita a previsão, e de forma coerente com ela, o grupo planeja e antecipa o que fará. Ao mesmo tempo, assistindo às participações de outras equipes, as novas informações subsidiam e ratificam o raciocínio desenvolvido.

Nesse sentido, o uso da HM de Planejamento ${ }^{(c)}$, de certa forma, levou ao estabelecimento de um diagnóstico que foi definido antes mesmo de saberem qualquer coisa sobre o caso real.

[Francisco] Eu e a Mariana a gente entrou com a cabeça de síndrome coronariana e a gente queria encaixar isso no paciente...

[Francisco] É acho que esse foi o que atrapalhou, porque a gente foi o terceiro caso, [...] e a gente sabia que se viesse uma síndrome coronariana, a gente sabia manejar bem, aí a gente estava preparado...

[Carlos] É, foi até o assunto que a gente teve no intervalo de troca do caso e aí a gente pegou e revisou o que cada um sabia[...]

É importante ressaltar que inicialmente, no cenário do TEP, o diagnóstico de IAM era também possível. Os primeiros dados permitiam essa hipótese diagnóstica.

O cenário inicial pôde então ser interpretado como previsto originalmente. É, portanto, o momento em que a Experiência Metacognitiva $^{(\mathrm{d})}(\mathrm{EM})$ de Familiaridade ${ }^{(\mathrm{e})}$ elicita a EM de Sentimento de Confiança ${ }^{(f)}$ (SC). Por isso, a equipe sente-se confortável em prosseguir no atendimento, mesmo tendo em mãos um diagnóstico inadequado.

[Francisco] Aí chegou um caso pra gente de uma paciente com dor torácica...

[Mariana] Eu acho que, síndrome coronariana pelo menos, foi o que eu mais vi assim em pacientes de verdade... e você pensa, dor torácica, tosse às vezes têm, mas não é associado, então você acaba gravando mais isso por ser mais comum, mais frequente no atendimento.

[Francisco] Aí foram os quatro e a gente já começou investigar para isso e queria encaixar de qualquer jeito aqui, porque o diagnóstico diferencial não muda...

Até esse ponto, embora enviesado, o processo de pensamento ocorre sem que a equipe perceba maiores dificuldades. Baseada no pressuposto de um cenário de IAM, a equipe, mesmo omitindo aspectos relevantes ao diagnóstico correto, vai confirmando o diagnóstico original ao privilegiar 
os sinais e sintomas enviesadamente selecionados. Isso gera na equipe um Sentimento de Confiança generalizado, que retroalimenta tanto o diagnóstico original quanto os procedimentos terapêuticos subsequentes.

Como afirmam Terra-Filho et $\mathrm{al}^{17}$. "Devido ao seu quadro clínico multifacetado e à sua natureza muitas vezes silenciosa, o TEP é uma doença ainda enigmática do ponto de vista epidemiológico (p.4-5)", constituindo-se um importante problema de saúde no Brasil. Entre 1989 e 2010, registraramse aproximadamente 92 mil casos fatais, dos quais $55,8 \%$ eram mulheres, sendo a taxa de mortalidade padronizada por idade em 2010 de 2,62/100 mil (p.5) ${ }^{18}$. Ressalte-se aqui que o diagnóstico do TEP não é simples nem imediato. Nos quadros agudos, o diagnóstico diferencial inclui tamponamento cardíaco, IAM, dissecção aórtica, disfunção valvular aguda e hipovolemia ${ }^{19}$. Estima-se que, nos Estados Unidos, seja a terceira maior causa de morte em pacientes hospitalizados, e, apesar disso, a maioria dos casos não é diagnosticada em vida. $\mathrm{E}$ ainda mais, menos de $10 \%$ dos casos fatais receberam tratamento específico para a moléstia ${ }^{20}$. No Brasil, outros fatores que dificultam o diagnóstico são a superposição dos sinais e sintomas e também a inespecificidade de boa parte do quadro clínico ${ }^{21-24}$. A Figura 1 apresenta uma síntese desse contexto.

Naquele momento, como já mencionado, o IAM era uma possibilidade, porém não a única. Mas os primeiros achados foram reconhecidos como sendo indicativos apenas do IAM, pois o SC inibiu a HM de Detecção de Erro $^{(g)}(D E)$, o que permitiu o prosseguimento da conduta errada.

[Jaqueline] É e a gente que um dos casos, que era os casos mais comuns da experiência da prática, né?, vai ser síndrome coronariana, e a gente estava com a cabeça assim muito... e aí o diagnóstico foi, aí teve mais uma coisa, daí quando o eletro, quando parou e a gente analisou errado...

[Francisco] Tinha uma bradicardia sinusal mas...

Dessa forma, como a equipe não foi capaz de monitorar o ambiente, forneceu informação enviesada para o metanível ${ }^{(\text {h) }}$, dificultando a detecção do erro e, portanto, inibindo a HM de Avaliação(i), que poderia corrigi-lo. Ou seja, faltou monitorar e controlar o ambiente para verificar se a previsão se confirmava.

[Mariana] Não encaixa no que a gente estava pensando, mas o que mais a gente pode pensar que a gente está pulando?

[Jaqueline] E mesmo dentro do caso, a gente acabou omitindo o raio x que mudaria o diagnóstico.

[Mariana] A única dificuldade foi a falta de resposta que a gente estava focado. E o erro foi não parar e pensar.
[Jaqueline] É que a gente viu uma parte do diagnóstico, focou num diagnóstico e a gente esquece das queixas totais assim da paciente.

[Carlos] Naquele momento, a gente estava acreditando que era uma síndrome coronariana $e$ estava indo, a tosse e o fato da paciente ser fumante era uma coincidência...

[Francisco] E não passou pela cabeça de nenhum dos quatro o TEP...

[Jaqueline] Foi assim, foi não parar e, reanalisar o caso, a gente foi indo assim e indo nos nossos erros, foi errando e foi indo no nosso erro. A gente não parou e reavaliou a situação e ... a gente parou assim no final e falou assim: "Meu, não, vamos pensar no que está acontecendo". Aí já era tarde demais e já acabou a cena.

Em medicina, não se prossegue no erro impunemente. $A$ evolução negativa do quadro mostra a fragilidade do SC prévio. As respostas inadequadas da paciente (na perspectiva do que a equipe esperava) reduzem a confiança anterior, quando então aflora a EM de Sentimento de Dificuldade ${ }^{(j)}$.

\section{[Jaqueline] Até o momento que o paciente não evoluía bem aí...}

[Carlos] O paciente evoluiu com bradicardia e ... e daí encerrou o cenário.

[Jaqueline] Só que a gente não estava tão... "porque ele estava respondendo direito o que era para responder"; em vez de analisar o que estava acontecendo, a gente ficou: "Nossa, mas ele não está respondendo"...

[Francisco] A gente não expressou claramente isso, mas só que, no final da cena, a gente tinha certeza de que não era síndrome coronariana e era uma incógnita assim... nos momentos opostos assim a gente não sabia o que pensar.

A situação era de dificuldade e de falta de resposta da paciente à terapia prescrita. Para a equipe, isso estava claro. Mas, apesar disso, a equipe persistiu, buscando apenas os dados que confirmassem o diagnóstico original de IAM e distorcendo as HM de Monitoração(k) e Controle ${ }^{(1)}$, resultando, assim, na impossibilidade do uso das estratégias de DE e Resolução de Conflito $^{(m)}(R C)$ pela ativação da Memória de Trabalho ${ }^{(n)}(M T)$. Dito de outra forma, a equipe não parou de fazer o que fazia para tentar ver e resolver o que estava errado. E, aqui, uma solução inicial teria sido buscar na memória que outros diagnósticos diferenciais poderiam explicar o quadro atual.

Assim é que a equipe não transforma o Sentimento de Dificuldade em ferramenta de correção. E isso se dá por dois motivos. Por um lado, em termos práticos, por conta do encerramento do caso por decisão da banca julgadora. Por 


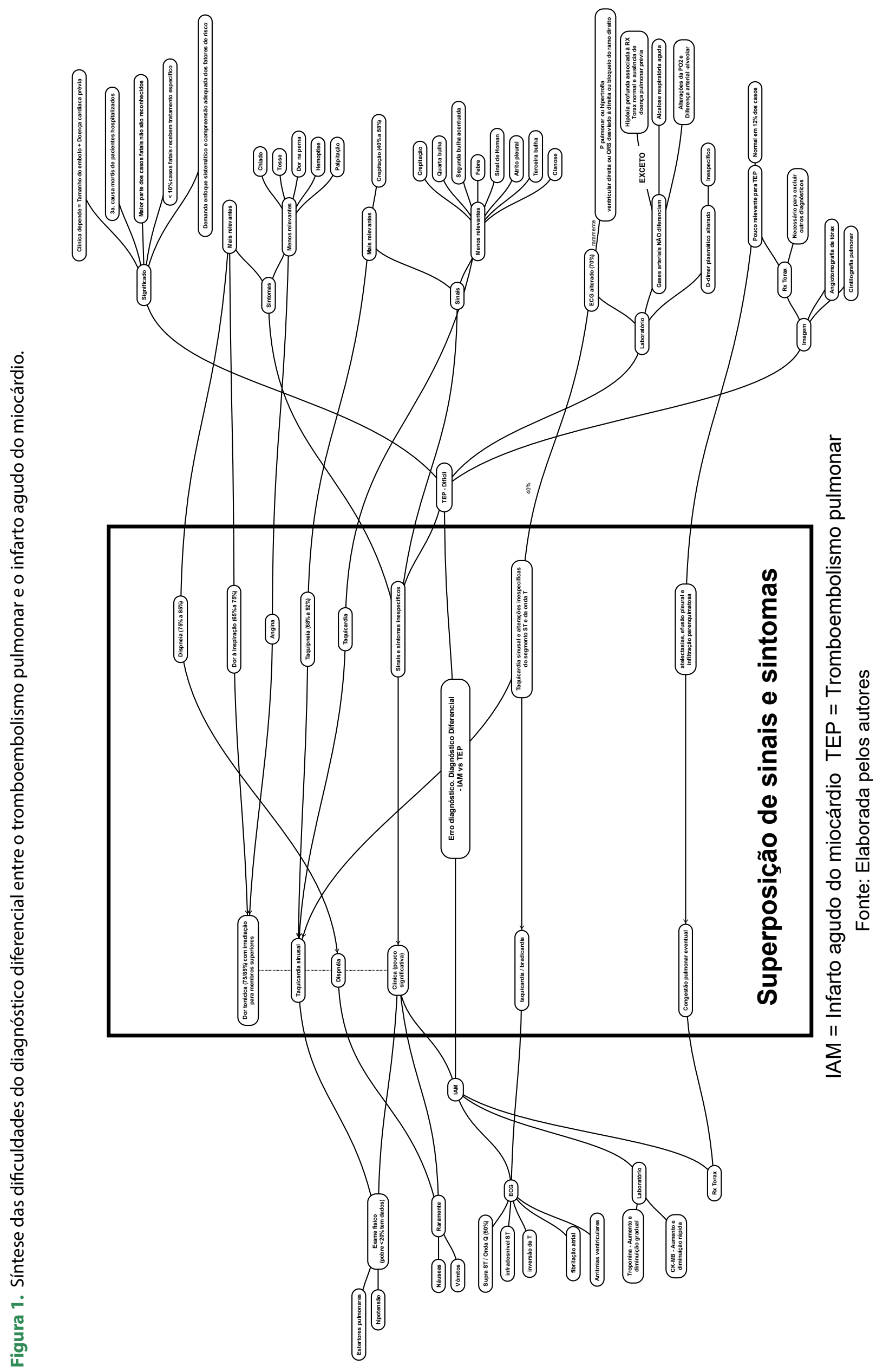


outro, esse "congelamento" na dificuldade pode ser explicado pelo fato do controle inadequado continuar enviesando a observação; impedindo a detecção do erro e a resolução do conflito entre a expectativa da equipe e a evolução do paciente. Conflito que poderia ter sido resolvido, como já mencionado, pela ativação da MT.

[Carlos] Teve um momento que a gente até comentou que, quando a gente tomou a decisão de entubar a paciente, ela insistiu que "ah não é porque eu sou fumante, isso não interfere?" e bateu três vezes no fato de ser fumante, aí eu parei e pensei: "Não, mas o fumo e a dor torácica por uma síndrome coronariana ela vai responder e não vai...." Então foi nesse momento que parei, pensei e isolei essa questão da tosse e do tabagismo, mas essa foi o que marcou pra mim assim, da insistência, parecia que tinha alguma coisa que puxava para isso, pra eu prestar atenção no tabagismo...

[Carlos] Eu percebi que eu tinha que prestar atenção no tabagismo, ... mas eu não achava em lugar nenhum e eu ignorei.

[Mariana] Parecia que estava sobrando...

[Jaqueline] Mas ficou um incômodo, a gente saiba que faltava uma peça, ... assim...

[Mariana] É tipo, o quebra-cabeça está completinho, mas e esse tabagismo, e essa tosse...

[Entrevistador] Mas havia esse sentimento de incômodo, de alguma forma?

[Jaqueline] Havia, sim, não só depois quando acabou, havia sim. Porque os exames vieram normais, não estava encaixando, só que na hora assim, eu acho que foi o...

[Francisco] Tinha o nervosismo também no primeiro caso...

[Jaqueline] É, esse foi, no primeiro caso foi o que mais impactou na gente foi o nervosismo, a gente não conseguia raciocínio, acho que na hora, foi muito nervosismo, não estava preparada assim...

Por decisão da banca julgadora, o cenário foi encerrado. Se houvesse mais tempo, esta narrativa poderia ter tido um desenlace melhor? É difícil saber, mas há indícios positivos. Durante todo o processo, houve vários sinais e sintomas que poderiam ter conduzido ao diagnóstico correto, mas que, pelas razões já expostas, foram eliminados da linha principal de raciocínio. No entanto, eles foram se somando e gerando um estado de incerteza e inquietude. $E$, finalmente, houve um quadro de bradicardia, que funcionou como incidente crítico para possível correção de rumo.

Como mencionado, a equipe iniciou o processo com um diagnóstico preconcebido e assim se manteve durante a maior parte do tempo, com as já descritas distorções e enviesamentos nas variáveis metacognitivas. $E$ a percepção do erro não se fez de forma instantânea. Ao longo do tempo, a equipe foi construindo vaga e imprecisamente essa percepção. Durante esse período, várias condições e dados foram se acumulando $\{*\}$, sendo expressos sob a forma do Sentimento de Dificuldade. O ápice desse processo foi a ocorrência da bradicardia (incidente crítico), que se configurou como a condição eficaz e instantânea $\left\{{ }^{* *}\right\}$ para atentar para a existência do erro. Nesse momento, a banca examinadora decidiu encerrar o cenário $\left\{*^{* *}\right\}$ impedindo as eventuais ações e pensamentos decorrentes do incidente crítico.

$\{*\}$ [Francisco] Eu acho que a partir do momento que o dado de anamnese... Não vinha achado de eletro que a gente gostaria que viesse, dados laboratoriais que a gente gostaria que viesse, talvez uma dor típica que a gente gostaria que viesse, desde o começo, dicas que diziam pra gente e que não era... $\left\{^{* *}\right\}$ É isso foi decisivo [a bradicardia] mas, $\{*\}$ a partir do momento de dados de anamnese, de exames que a gente solicitava não batiam com o que a gente estava pensando... $\left\{{ }^{*}\right\}$ Mas essa dificuldade que fez com que a gente continuasse investigando coisas que...

[Mariana] $\{*\}$ E pedimos todos os exames a mais.

[Entrevistador] Havia alguns sinais que vocês percebiam e foram se somando...

[Todos] $\left\{^{* *}\right\}$ Até o ponto da bradicardia.

[Mariana] $\left\{{ }^{*}\right\}$ Eu pelo menos, $\{*\}$ alguma angústia assim: "Eu estou fazendo o que eu sei. O que não está funcionando? O que eu estou fazendo de errado?".

[Carlos] $\left\{{ }^{* *}\right\}$ O momento decisivo foi a bradicardia que não resolveu e a partir daquele momento que a gente falou: "Não, tá errado".

[Mariana] $\left\{{ }^{*}\right\}$ Entrou a bradicardia...

[Francisco] $\{* *\}$ Foi isso que levou no momento a gente ter a percepção...

[Carlos] $\{* *$ \} $O$ paciente evoluiu com bradicardia $e$ [inaudível 19min46s] e daí encerrou o cenário.

Para concluir esta narrativa, imaginemos outro desenlace, em que hipoteticamente o erro seria corrigido por meio desta sequência de ações e raciocínios:

1. A bradicardia demanda a HM de Atenção Seletiva ${ }^{(0)}$ para diagnósticos diferenciais.

2. Para tal, ativa-se o Controle para monitorar o cenário, inibindo as ações atuais pelo Controle Inibitório(p) e reorganizando o quadro de modo que a Memória de Trabalho pudesse fornecer diagnósticos diferenciais 
para a Resolução de Conflito entre:

2.1. os dados originalmente indicativos da síndrome coronariana e outros dados que pudessem fazer o diagnóstico diferencial e

2.2. respostas do paciente e outras condições ambientais.

Até o momento, na perspectiva metacognitiva, o que aqui se descreveu foi o processo de pensamento subjacente às ações. Cabe agora contextualizar este estudo na perspectiva mais ampla do ensino médico. Já apontamos a simulação realística como metodologia ativa ${ }^{12}$. Cabe também situá-la no domínio das metodologias problematizadoras, discriminando-a da aprendizagem baseada em problemas ${ }^{6}$. Neste estudo, aproximamo-nos do primeiro caso, já que, na simulação, o contexto busca espelhar a realidade, tanto no que se refere ao ambiente físico e social, como também no conteúdo específico a ser trabalhado. Ademais e principalmente, porque o problema aflora do cenário real. No dizer de Maia ${ }^{25}$ :

No caso da problematização da realidade, um recorte do mundo (real ou, ao menos, verossímil) desencadeia a aprendizagem do aluno, por aproximá-lo do estudo de uma situação clínica real, e não de um tema ou conteúdo cuja aplicabilidade em sua prática não esteja claramente situada. Não se trata simplesmente de um acréscimo de saberes aos anteriormente construídos, mas do estabelecimento de novas e eventualmente complexas redes entre conceitos aprendidos (p.569).

Muito recomendado nesses casos é o Arco de Maguerez, orientador de um ciclo pedagógico que tem na realidade seu ponto de partida e chegada, onde entre um e outro o aluno observa, seleciona os aspectos relevantes, recorre à teoria e cria hipóteses de solução, que são finalmente testadas na realidade. Dessa forma, então, é possível retornar à teorização, só que agora mais densa e fundamentada no real.

Supomos haver forte superposição entre o arco e os processos de simulação, quando se substitui o termo "realidade" por "cenário". Assim, o estudante em uma simulação precisa observar o cenário, identificar o problema e discriminar o irrelevante do incidental. Sozinho, ou com o apoio do professor, interage com a teoria para a construção das hipóteses diagnósticas e as testa, recorrendo aos exames complementares ou, em fase posterior, aplicando as terapias pertinentes.

Ao final do processo, durante o debriefing, novamente acessa a teoria, agora em condição mais complexa e completa, na medida em que seus conhecimentos prévios integraramse aos fornecidos pelo professor e aos oriundos do cenário simulado, tornando-se mais conscientes pelo próprio processo do debriefing.

Nesse entendimento, o domínio do Arco de Maguerez é o cognitivo. Professor e aluno debruçam-se ora sobre o ambiente externo (o paciente, o sistema de saúde etc.), ora sobre o conteúdo cognitivo, sob a forma de fatos e conceitos médicos na etapa de teorização. Além disso, sua utilização se faz por um processo de interação entre professor e aluno, que tem por objetivo promover a aprendizagem significativa ${ }^{6}$. Na Teoria da Subsunção de David P. Ausubel, o significado de um conceito se constitui não apenas na sua definição, mas também e principalmente na sua relação com todos os outros que com ele se relacionam. Assim, é pouco importante que um aluno apenas saiba definir o que é hipovolemia. Mais significativo é que ele seja capaz de fazê-lo no contexto da fisiopatologia do choque, por exemplo. E é apenas quando o aprendizado de um novo conceito não se faz pela mera adição à trama, mas pela sua reorganização, é que a aprendizagem significativa ocorre ${ }^{7,8}$.

Por isso, o Arco de Maguerez se afina tão bem com a teoria ausubeliana. Nas várias etapas de observação, construção de hipóteses, teorização e testes, constitui-se como importante instrumento de operacionalização no ensino médico da mencionada teoria.

Quando tratamos de um domínio metacognitivo, abordamos a capacidade de gerenciar o pensamento. $E$, ainda mais, como a metacognição pode contribuir para o ensino médico e, especificamente aqui, para o desenvolvimento de competência profissional. E para fins deste estudo, o termo é entendido como "capacidade circunstancial de mobilizar articuladamente os recursos cognitivos, psicomotores e afetivos, visando abordar ou resolver uma situação complexa (p.205)" ${ }^{\prime \prime}$.

A metacognição é mais do que uma descrição simples do processo cognitivo. Ela possui a capacidade de gerenciar esse processo conforme postula o modelo de Nelson et al. ${ }^{26}$. Para os autores, o pensamento ocorre em dois níveis: cognitivo e metacognitivo. Neste último, localizam-se os modelos ideais de funcionamento, e é ele que monitora o nível cognitivo. Por meio de mecanismos de monitoração, o nível cognitivo informa ao nível metacognitivo a evolução dos processos, e este avalia sua adequação e controla o cognitivo, indicando a manutenção ou as alterações dos processos em curso.

Observe-se que o que une esses três domínios é o pensamento ativo e reflexivo. Énecessário pensar na prática com o Arco de Maguerez. Aprender significativamente é relacionar conceitos, e ser competente é fazer escolhas deliberadas. Govaerts" ${ }^{27}$, inclusive, é explícito ao afirmar que "saber não é suficiente para fazer, assim como fazer não é suficiente para aprender: competência requer experiência e reflexão na prática profissional em qualquer nível de experiência (p.235)".

\section{CONCLUSÃO}

A presente pesquisa contribui para a literatura da educação médica ao fornecer um estudo de caso sobre 
os processos metacognitivos presentes em uma equipe de estudantes de Medicina que atende a uma simulação de emergência médica no contexto de uma "olimpíada de simulação de atendimento a pacientes críticos".

Foi possível demonstrar que o mero domínio do conteúdo específico não foi suficiente para resolver a situação complexa proposta. O diagnóstico médico adequado implica conhecimento médico suficiente e uso correto dos processos de raciocínio clínico. Aqui, aparentemente, o erro se deu não por deficiência de conhecimento prévio, mas pelo enviesamento do raciocínio. Isto é, a equipe fixou-se em uma perspectiva de confirmação, sem atentar para sinais e sintomas discordantes.

A primeira novidade deste estudo foi demonstrar, por meio da investigação metacognitiva, a possibilidade de tornar visível o processo. E, ainda mais, pelo menos neste caso, por ser capaz de explicitar como e por que ocorre um tipo específico de erro de raciocínio médico.

A segunda, decorrente da primeira, foi apontar a metacognição, pela sua capacidade de gerenciar o pensamento, como uma ferramenta didática importante, inclusive permitindo integrar o Arco de Maguerez e a aprendizagem significativa na busca pelo ensino médico para a competência. Assim, os dados coletados avançam no campo teórico propondo uma integração entre quatro aspectos: metacognição, Arco de Maguerez, aprendizagem significativa e desenvolvimento de competências.

Apesar de os autores terem ciência que, neste momento, a integração proposta, mesmo que com fundamentação, ainda é conjectural, eles consideram sua originalidade ao justificarem sua menção neste estudo. Entendem, ainda, que a proposta deve ser mais bem trabalhada, mas que tal escopo foge do foco e dos limites deste texto, estando destinada a ser mais bem cuidada em estudos futuros.

Por isso, os resultados desta pesquisa fornecem subsídios tanto para melhorar o design e a entrega de treinamento de simulação baseado em equipe, quanto para melhorar os processos de ensino e aprendizagem para estudantes e profissionais da área médica.

\section{NOTAS/GLOSSÁRIO}

a) Competências necessárias para o controle voluntário sobre seus próprios processos cognitivos por meio do conhecimento processual, expresso pelo uso deliberado das estratégias².

b) Habilidade de permitir o pensar sobre os objetivos de aprendizagem, as características adequadas de aprendizagem e o tempo disponível28.

c) Permite pensar com antecipação: como, quando e por que agir no sentido de obter os seus objetivos, por meio de uma sequência de submetas que conduz à meta principal do problema ${ }^{28}$.

d) Do que a pessoa está consciente e o que ela sente quando encontra uma tarefa e processa as informações relacionadas a ela ${ }^{5}$. Trata-se da interface entre a pessoa e a tarefa.

e) É uma informação (afetiva) sobre a ocorrência anterior de um estímulo. Denota a fluência de processamento e está associado a afetos positivos decorrentes da fluência na acessibilidade da respectiva informação².

f) É informação que aflora ao final do processamento de uma tarefa, resultante do balanceamento entre os sentimentos positivos e negativos percebidos pelo indivíduo sobre essa tarefa ${ }^{29}$.

g) A HM de Detecção de Erro é entendida como identificação de discrepâncias entre o objetivo e o produto do processamento, é frequentemente considerado um índice de monitoramento da compreensão ${ }^{30}$.

h) Modelo teórico de funcionamento da metacognição, composto por dois níveis, o metanível e o nível objeto, mantendo, entre si, constante fluxo informacional. No nível objeto, os eventos cognitivos acontecem, tais como o aprendizado, a memorização, etc. Já o metanível contém um modelo dinâmico do nível objeto, monitorando-o continuamente para, sempre que necessário, controlá-lo inibindo, iniciando ou modulando uma ação cognitiva ${ }^{26}$.

i) Integra o Conhecimento e o Sentimento Metacognitivos para definir estado atual do resultado e curso futuro do processamento da tarefa. É uma monitoração da fonte. Nessas tarefas, os indivíduos devem avaliar informações contextuais, tais como lembrar-se de quando ou onde ocorreu algum evento ou quem apresentou alguma informação ${ }^{31}$.

j) Alerta o indivíduo sobre a necessidade de tomada de decisão porque há conflito de respostas e aumento da probabilidade de erro 32 .

k) A HM de Monitoração é definida como o controle autorregulado de habilidades cognitivas usadas durante o desempenho atual, a fim de identificar problemas $e$ modificar planos ${ }^{28}$.

l) Sistema de metanível que atua sobre os processos cognitivos de nível mais baixo. É, no sentido do fluxo informacional, um processo de baixo para cima, como a atribuição de mais tempo de estudo ou o lançamento de algumas estratégias de recuperação ${ }^{26}$.

m) Refere-se a processos que coordenam a cognição a partir das informações fornecidas pelo processo de controle ${ }^{33}$.

n) A Memória de Trabalho se refere aos processos e às representações envolvidos na ativação temporária ou no 
armazenamento de informação ${ }^{34}$.

o) Em tarefas com mensagens competitivas, o indivíduo é solicitado a selecionar uma informação e ignorar as demais, e, portanto, deverá recuperar somente uma das informações. Refere-se à habilidade de inibir respostas competitivas e irrelevantes ${ }^{30}$.

p) É a capacidade de adiar, focar a atenção e reprimir desejos imediatos ou impulsos. Relaciona-se de maneira próxima a traços considerados como prototípicos da capacidade de restrição: deliberação, controle de impulsos, capacidade de planejar e persistência na consecução de metas distante ${ }^{35}$.

\section{CONTRIBUIÇÃO DOS AUTORES}

Mauricio Abreu Pinto Peixoto participou da concepção da pesquisa, do planejamento e da definição de técnicas e métodos, do trabalho de campo, do processamento e da análise dos dados, do levantamento da literatura, da construção do texto e da revisão e formatação final. Marcos Antônio Gomes Brandão participou da concepção da pesquisa, da construção do texto e da revisão e formatação final. Gerson Alves Pereira Junior participou da concepção da pesquisa e da revisão e formatação final. Juliana Faria Campos participou do levantamento da literatura, da construção do texto e da revisão e formatação final. Jaqueline da Silva Soares Souto participou da construção do texto e da revisão e formatação final.

\section{CONFLITO DE INTERESSES}

Os autores declaram não haver conflito de interesses neste estudo.

\section{FINANCIAMENTO}

Fundação Carlos Chagas Filho de Amparo à Pesquisa do Estado do Rio de Janeiro - Jaqueline da Silva Soares Souto: Bolsista Faperj n²00.839/2019 (242621).

\section{REFERÊNCIAS}

1. Brydges R, Butler D. A reflective analysis of medical education research on self-regulation in learning and practice. Med Educ. 2012;46:71-9.

2. Zimmerman BJ, Schunk DH, organizadores. Handbook of self-regulation of learning and performance. New York: Routledge; 2011. 484 p.

3. Flick U. Entrevistas. In: Caregnato SE, organizador. Uma introdução à pesquisa qualitativa. 3a ed Porto Alegre: Bookman; 2009. p. 143-79.

4. Bardin L. Análise de conteúdo. 3a reimp. São Paulo: Edições 70; 2011. v. 1, $280 \mathrm{p}$.

5. Efklides A. Metacognition-defining its facets and levels of functioning in relation to self-regulation and co-regulation. Eur Psychol. 2008;13(4):277-87.

6. Tsuji H, da Silva H. A. Aprender e ensinar na escola vestida de branco: do modelo biomédico ao humanístico. São Paulo: Phorte; 2010. 240 p.

7. Ausubel DP. Educational psychology: a cognitive view. New York. Holt, Rinehart and Winston; 1968. 685 p.

8. Moreira MA. Aprendizagem significativa: a teoria e textos complementares. São Paulo: Livraria da Física; 2011. 179 p.
9. Norman GR, Monteiro SD, Sherbino J, Ilgen JS, Schmidt HG, Mamede S. The causes of errors in clinical reasoning: cognitive biases, knowledge deficits, and dual process thinking. Acad Med. 2017;92(1):23-30.

10. Bond WF, Deitrick LM, Arnold DC, Kostenbader M, Barr GC, Kimmel SR, et al. Using simulation to instruct emergency medicine residents in cognitive forcing strategies. Acad Med. 2004;79(5):438-46.

11. Manser T, Foster S. Effective handover communication: an overview of research and improvement efforts. Best Pract Res Clin Anaesthesiol. 2011;25(2):181-91.

12. Mazzo A, Almeida RG, Batista RCN, Pedersoli E, Girão FB, Martins JCA. Simulação: conceitos básicos. In: Scalabrini Neto A, Fonseca AS, Brandão CFS, organizadores. Simulação realística e habilidades na saúde. Rio de Janeiro: Atheneu; 2017. p. 49-57.

13. Duffy MC, Azevedo R, Sun N-Z, Griscom SE, Stead V, Crelinsten L, et al. Team regulation in a simulated medical emergency: an in-depth analysis of cognitive, metacognitive, and affective processes. Instr Sci. 2015;43(3):401-26.

14. Greenberg SB, Tokarczyk A, Small S. Critical care simulation. Dis Mon. 2011;57(11):715-22

15. McGaghie WC, Issenberg SB, Petrusa ER, Scalese RJ. A critical review of simulation-based medical education research: 2003-2009: simulationbased medical education research 2003-2009. Med Educ. 2010;44(1):50-63.

16. Peixoto MAP, Brandão MAG, Santos G. Metacognição e tecnologia educacional simbólica. Rev Bras Educ Med. 2007;31(1):67-80.

17. Terra-Filho $M$, Menna-Barreto SS. Recomendações para o manejo da tromboembolia pulmonar, 2010. J Bras Pneumol. 2010;36(supl 1):1-3.

18. Darze ES, Casqueiro JB, Ciuffo LA, Santos JM, Resedá I, Latado AL. Mortalidade por embolia pulmonar no Brasil entre 1989 e 2010: disparidades regionais e por gênero. Arq Bras Cardiol. 2016;1:4-12.

19. Konstantinides SV, Meyer G, Becattini C, Bueno H, Geersing G-J, Harjola V-P, et al. 2019 ESC Guidelines for the diagnosis and management of acute pulmonary embolism developed in collaboration with the European Respiratory Society (ERS). Eur Heart J. 2020;41(4):543-603.

20. Chesnutt MS, Prendergast TJ. Pulmonary venous thromboembolism In: Tierney Jr. LM, McPhee SJ, Papadakis MA, editors Current medical diagnosis \& treatment. 44th ed. New York : LangeMedical Books/McGrawHill; 2005. p. 276-88.

21. Caramelli B, Gottschall CAM, Blacher C, Casagrande EL, Lucio EA, Manente ERF, et al. Diretriz de embolia pulmonar. Arq Bras Cardiol. 2004;83:1-8.

22. Borrayo-Sánchez G, Pérez-Rodríguez G, Martínez-Montañez OG, AlmeidaGutiérrez E, Ramírez-Arias E, Estrada-Gallegos J, et al. Protocol for the care of acute myocardial infarction in emergency: Código infarto (The Infarction Code). Rev Med Inst Mex Seguro Soc. 2017;55(2):233-46.

23. Hospital Sírio-Libanês. Protocolo Sírio-Libanês: tromboembolismo venoso e pulmonar [acesso em 27 mar 2019]. Disponível em: https:// www.hospitalsiriolibanes.org.br/institucional/gestao-da-qualidade/ Documents/2018-11-01-protocolos/Protocolo\%20TEV/Protocolo\%20 TEV_VF.pdf.

24. Piegas L, Timerman A, Feitosa G, Nicolau J, Mattos L, Andrade M, et al. V Diretriz da Sociedade Brasileira de Cardiologia sobre Tratamento do Infarto Agudo do Miocárdio com Supradesnível do Segmento ST. Arq Bras Cardiol. 2015;105(2):1-121. doi: 10.5935/abc.20150107.

25. Maia JA. Metodologias problematizadoras em currículos de graduação médica. Rev Bras Educ Med. 2014;38(4):566-74.

26. Nelson, T. O.; Narens, L.. Why Investigate Metacognition? In: J. Metcalfe; A. P Shimamura (Orgs.); Metacognition. p.1-25, 1994. The MIT Press. Disponível em: <https://direct.mit.edu/books/book/3931/chaPVTEr/164549/whyinvestigate-metacognition>. Acesso em: 11/4/2021.

27. Govaerts MJB. Educational competencies or education for professional competence? Med Educ. 2008;42(3):234-6.

28. Desoete A. Multi-method assessment of metacognitive skills in elementary school children: how you test is what you get. Metacogn Learn. 2008;3(3):189-206. 
29. Efklides A, Petkaki C. Effects of mood on students' metacognitive experiences. Learn Instr. 2005;15:415-31.

30. Shimamura AP. The role of the prefrontal cortex in dynamic filtering. Psychobiology. 2000;28(2):207-18.

31. Johnson MK, Hashtroudi S, Lindsay DS. Source monitoring. Psychol Bull. 1993;114(1):3-28.

32. Veen $\mathrm{V}$ van, Carter CS. The timing of action-monitoring processes in the in the anterior cingulate cortex. J Cogn Neurosci. 2002;14(4):593-602.
33. Fernandez-Duque D, Baird JA, Posner MI. Executive attention and metacognitive regulation. Conscious Cogn. 2000;9(2):288-307.

34. Shimamura AP. Toward a cognitive neuroscience of metacognition. Conscious Cogn. 2000;9(2):313-23.

35. Kochanska G. Multiple pathways to conscience for children with different temperaments: from toddlerhood to age 5. Dev Psychol. 1997;33(2):228-240. 